This item was submitted to Loughborough's Research Repository by the author.

Items in Figshare are protected by copyright, with all rights reserved, unless otherwise indicated.

\title{
Adaptive diurnal prediction of ambient dry-bulb temperature and solar
} \section{radiation}

PLEASE CITE THE PUBLISHED VERSION

PUBLISHER

(c) American Society of Heating, Refrigerating and Air-Conditioning Engineers, Inc.

LICENCE

CC BY-NC-ND 4.0

\section{REPOSITORY RECORD}

Ren, Mei J., and Jonathan A. Wright. 2019. "Adaptive Diurnal Prediction of Ambient Dry-bulb Temperature and Solar Radiation". figshare. https://hdl.handle.net/2134/3719. 
This item was submitted to Loughborough's Institutional Repository (https://dspace.lboro.ac.uk/) by the author and is made available under the following Creative Commons Licence conditions.

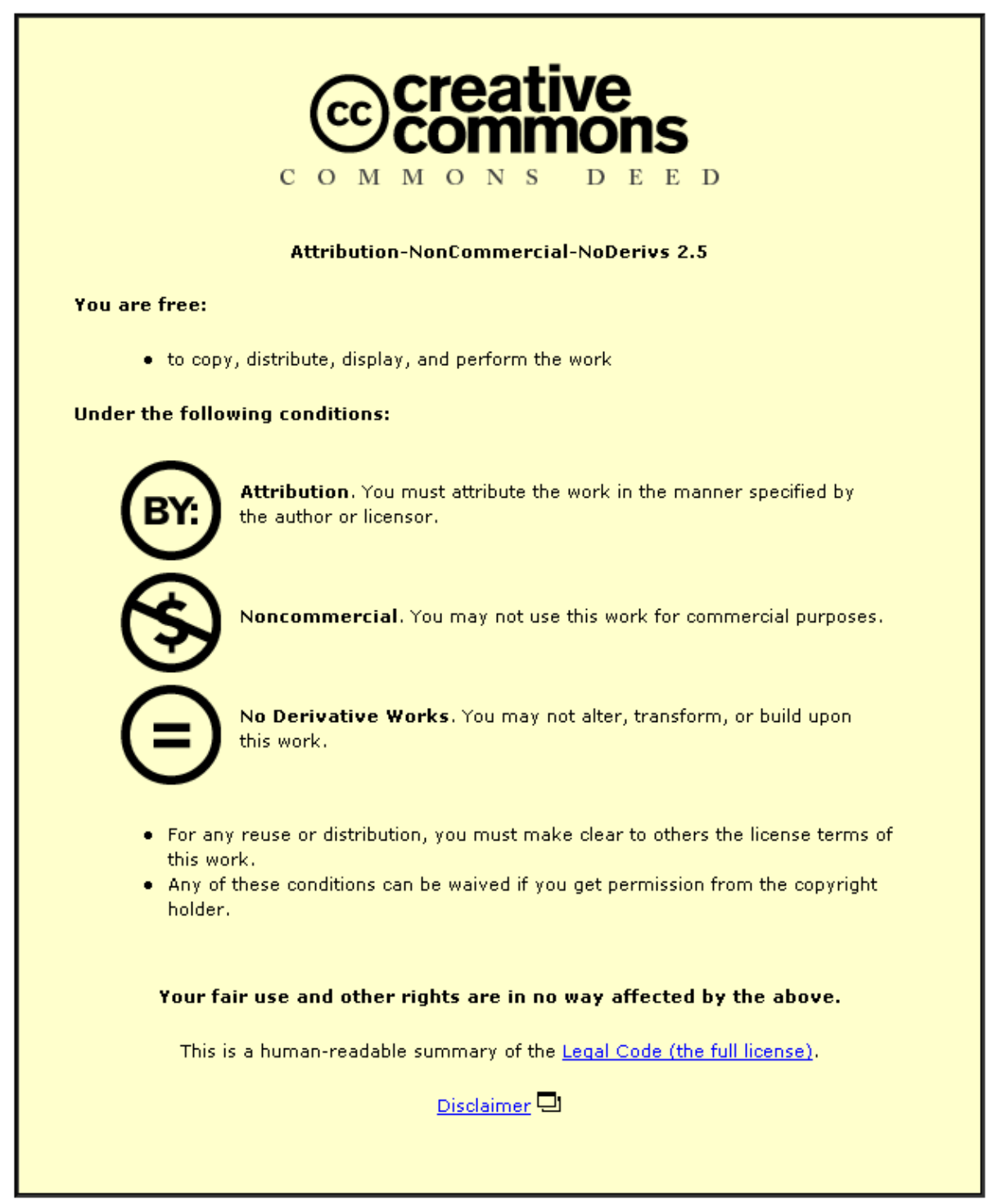

For the full text of this licence, please go to: http://creativecommons.org/licenses/by-nc-nd/2.5/ 


\title{
Adaptive Diurnal Prediction of Ambient Dry-Bulb Temperature and Solar Radiation
}

M.J. Ren

\author{
J.A. Wright \\ Member ASHRAE
}

\begin{abstract}
This paper presents a new adaptive weather-prediction model that can be used for on-line control of HVAC and thermal storage systems. The model can predict external dry-bulb temperature and solar radiation over the next $24 \mathrm{~h}$. Because a building with a fabric thermal storage system has a slow response to thermal loads, a predictive controller is essential to operate the building and associated plant installation to respond effectively to external climatic conditions ahead of time. Three prediction methods are investigated in the paper: a pure stochastic method, a combined deterministic-stochastic method, and an expanded method for short-term temperature forecast. It has been found that the combined deterministic-stochastic method is simpler and gives the smallest prediction errors. For the prediction of solar radiation, a deterministic model is proposed. The proposed prediction algorithms for temperature and radiation are simple and efficient to conduct on a supervisory PC to predict hourly temperature and radiation profiles over the next $24 \mathrm{~h}$. Updating temperature forecasts using observations available with time is also investigated in this paper.
\end{abstract}

\section{INTRODUCTION}

Current trends toward energy-efficient buildings have led to research into the optimum control of HVAC systems that use the building fabric as a thermal store. Two systems have been investigated: one for conventional air-conditioned buildings where fabric thermal storage is achieved by exposing the building fabric to the internal environment (Keeney and Braun 1997), and the other for buildings that use ventilated floor slabs, the ventilation air providing greater thermal coupling with the building fabric (Ren 1997). In both systems, the schedule of control set points is predicted to give the optimum system performance with minimized energy cost over a period of $24 \mathrm{~h}$. To schedule the control set points to make use of free night cooling and lower electricity costs, it is necessary to predict the weather conditions over the next $24 \mathrm{~h}$. The performance of such a control optimizer has been investigated (Ren 1997). This paper presents an approach to predicting the hourly ambient dry-bulb temperature and solar radiation conditions over a $24 \mathrm{~h}$ period. The predicted weather information is then used as the basis for the building simulation and optimization of control set-point scheduling. An application of the weather prediction model in a predictive controller was described in Ren and Wright (1997). Updating weather prediction hourly is also investigated in this paper.

\section{BACKGROUND}

External climatic conditions such as outdoor air temperature and solar radiation vary with time, which results in fluctuating heating and cooling loads in the building space. Most previous

Mei J. Ren is a senior specialist engineer with Buro Happold Engineering, Manchester, United Kingdom. Jonathan A. Wright is a senior lecturer in the Department of Civil and Building Engineering, Loughborough University, United Kingdom. 
research requiring weather modeling has focused on the development of predictive algorithms for heating and cooling loads directly rather than the ambient weather conditions (Ferrano and Wong 1990; Forrester and Wepfer 1984; MacArthur et al. 1989; Rupanagunta et al. 1995; Seem and Braun 1991). In particular, Kawashima et al. (1995) compared autoregressive integrated moving average (ARIMA), exponential weighted moving average (EWMA), linear regressive (LR), and artificial neural network (ANN) models in prediction of thermal loads over $24 \mathrm{~h}$; the results indicated that the ANN model produced the lowest prediction errors. Weather models for use in building design load analysis have also been investigated. Jiang and Hong (1993) used a stochastic weather model to obtain diurnal changes in the climatic variables and shape factors to estimate the hourly values. Yoshida and Terai (1992) used an autoregressive moving average (ARMA) model, which took account of the deterministic annual and diurnal periodicity and the stochastic variations in the climatic variables, including the ambient air temperature, solar radiation, and absolute humidity. Among the methods investigated for predicting weather conditions for use in predictive control are stochastic dynamic models (Holst et al. 1987), sinusoidal functions (Athienitis 1988), and shape factors and look-up tables (Chen and Athienitis 1996).

Although much research has focused on weather prediction, or on the result of climatic conditions on building thermal loads, no research has been conducted addressing the prediction of ambient conditions for use in set-point scheduling control. The requirement and assumption made of such a prediction algorithm are that the climatic conditions relating to the buildings are measured hourly and are available to update the model prediction. The model is required only to predict the ambient conditions for the next $24 \mathrm{~h}$. The model need not address the annual periodicity or seasonal effect separately, because the forecasting is to be conducted daily, wherein the parameters of the model are updated to include the effect of the measured data from the previous $24 \mathrm{~h}$. Three adaptive models for the diurnal prediction of ambient temperature and solar radiation are examined here, each candidate model having been selected through an understanding of the properties of ambient temperature and solar radiation time series.

In this paper, two sets of weather data have been selected to analyze the performance of the prediction models. The U.K. Chartered Institution of Building Services Engineers (CIBSE) example weather year, measured in London, U.K., from October 1964 to September 1965, and the data measured in Garston, U.K., during 1994 were used. The CIBSE example year is considered suitable for use in predicting average building energy consumption, whereas the data from 1994 are useful for predicting the potential overheating risk in low-energy buildings. Clearly, these data are for a temperate climate, but one that can be subject to significant stochastic changes in climate, and therefore is potentially challenging to model.

A weather model is complicated by the fact that the climatic variables are correlated to each other. Yoshida and Terai (1990-1991) suggested that the model could be simplified if it is assumed that temperature is only correlated to solar radiation, but that solar radiation is not correlated to any other variables.

Figure 1 shows the profiles for the hourly mean and standard deviation of global radiation for the CIBSE example year and 1994 (diffuse and direct radiation have also been considered, but are not presented here). It is observed that the properties of the radiation data are time dependent and thus are a nonstationary stochastic time series. The global radiation has a strong periodicity; the radiation at night is deterministically zero, reaching the peak in the middle of the day. The standard deviations indicate a high variation of the hourly global radiation in the middle of day, the highest variation being $218 \mathrm{~W} / \mathrm{m}^{2}, 68.5 \%$ of the highest mean radiation $318 \mathrm{~W} / \mathrm{m}^{2}$. This implies a significant change in the hourly radiation throughout an entire year.

Figure 2 shows the profiles for the hourly mean and standard deviation of the ambient temperature for the CIBSE example year and 1994. It is observed that the temperature series are also time dependent and thus are a nonstationary stochastic time series. 

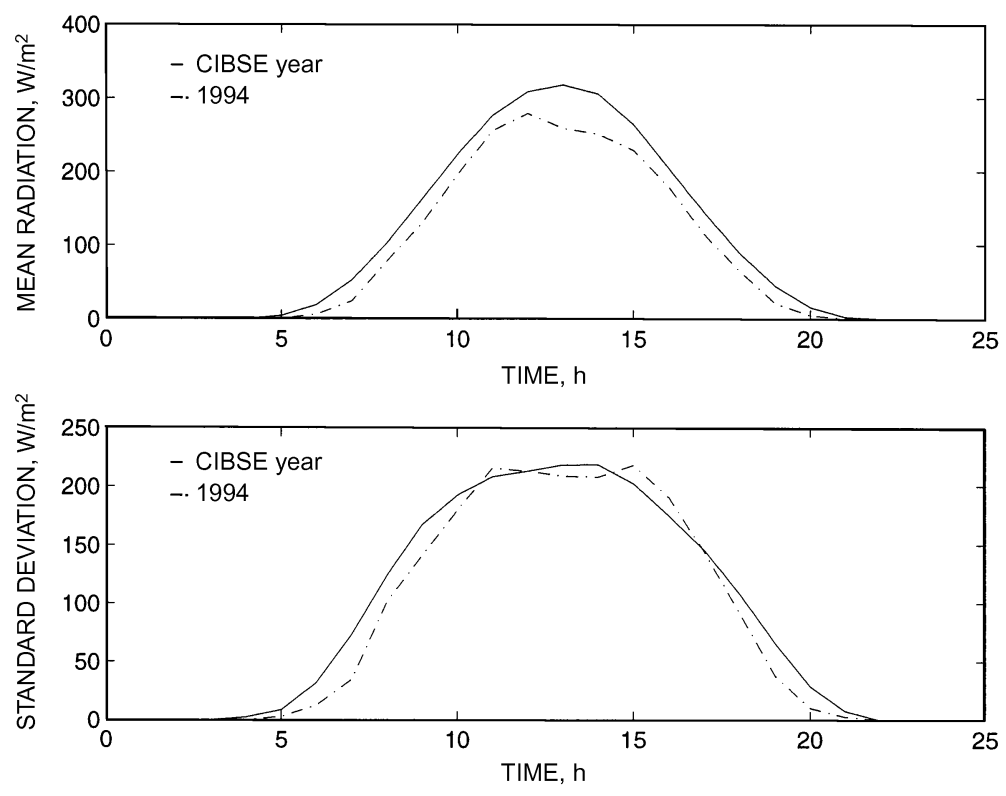

Figure 1. Hourly Mean and Standard Deviation of Global Radiation
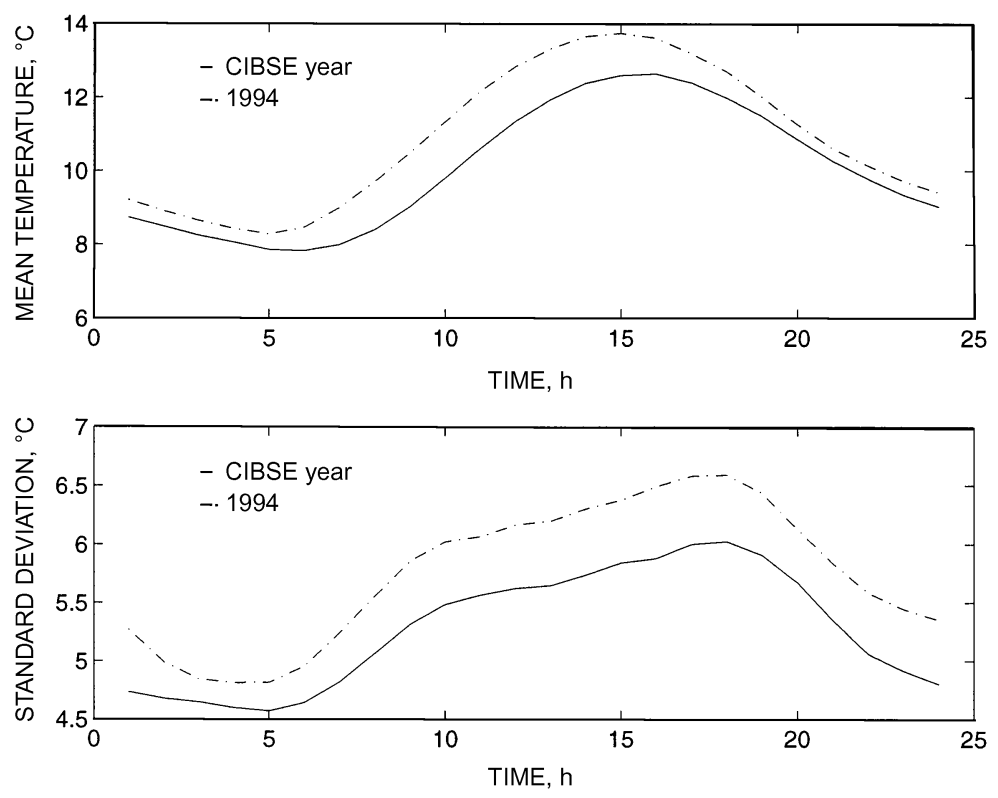

Figure 2. Hourly Mean and Standard Deviation of Ambient Temperature

The correlation between the ambient dry-bulb temperature and solar radiation (and the need to consider this in any modeling) has been investigated through the daily average temperature and daily total global radiation for the hourly example data. It was found that, over the entire year, there is a strong correlation between the two variables, with $\rho_{x y}{ }^{8}=0.5 \sim 0.6$ (correlation coefficient of $x$ and $y$, where $0=$ no correlation, $1=$ complete correlation) from the two sets of weather data. However, there is a much weaker correlation between the two variables when 
calculated for each month, which varies $\left(\rho_{x y}=0.08 \sim 0.6\right)$ depending on the month. This reflects the randomness of the two variables, especially the total solar radiation, which is greatly affected by cloudiness.

The high degree of randomness in the solar radiation data suggests that it may not be necessary to consider their correlation for short-term weather prediction when using recent historical data, as the influence of the solar radiation on the ambient temperature may have been reflected in the historical temperature itself. Therefore, the solar radiation and ambient temperature can be calculated independently. This will be justified further in the following investigation on the methods for modeling the temperature data. For a long-term weather prediction model, the influence of global solar radiation on the dry-bulb temperature may need to be taken into account, as in the method used by Yoshida and Terai (1990-1991).

\section{MODELING APPROACH}

Both the temperature and solar radiation data are nonstationary time series. Pandit and $\mathrm{Wu}$ (1983) claimed that an ARMA system can sufficiently model nonstationary time series if the model parameters are selected correctly, with the trend or periodicity exhibited in the data reflected in the roots of the parameters. However, by making the raw temperature time series stationary, the number of parameters of the ARMA model can be substantially reduced. This can be achieved by subtracting the deterministic component from the raw data using a deterministic model. The modeling methods used here to investigate the modeling of solar radiation and temperature time series include the following:

- Stochastic models comprised of single-variable ARMA models and multivariable autoregressive moving average with exogenous variables (ARMAX) models

- Deterministic models comprised of EWMA models and sinusoidal functions

The single-variable ARMA model has been applied to ambient temperature prediction, as has a combined deterministic and ARMA model (using both the EWMA and sinusoidal deterministic models). The multivariable ARMAX model has been used to investigate the need to include the solar radiation in the ambient temperature prediction. Prediction of solar radiation has been investigated using the combined stochastic-deterministic models and the deterministic models alone. Because this paper is concerned with the adaption of such models at least once a day, updating the model parameters is also discussed.

In most cases, hourly time step is used to forecast temperature and solar radiation profiles; the expanded combined stochastic-deterministic model, however, uses daily time step to predict the daily mean ambient temperature by using the daily total global radiation as one of the input data.

Hourly time step is adequate for supervisory control of a fabric thermal storage system, which is the main application of the weather prediction models investigated here. A time step shorter than an hour is unnecessary because of the thermal inertial of the system. For applications that may require shorter time intervals, such as 15 min or less, much simpler methods can be used such as simply using the previous temperature observations as the forecast for the present time step.

Four modeling errors have been used to evaluated the performance of the weather forecast models: the root mean square error (RMSE), the mean error (ME), the mean absolute error (MAE), and the maximum absolute error (MAXAE).

\section{ARMA Model}

An ARMA model uses stochastic linear difference equations to model the dependence of the data (Pandit and Wu 1983). The model can then be used to forecast the behavior of the system 
described by the data. Because the climatic data considered here are dependent or correlated between the observations, an ARMA model can be used to describe the dependence. The basic formula of an ARMA model is

$$
\hat{X}_{t}=\phi_{1} X_{t-1}+\phi_{2} X_{t-2}+\cdots+\phi_{n} X_{t-n}+a_{t}-\theta_{1} a_{t-1}-\theta_{2} a_{t-2}-\cdots-\theta_{m} a_{t-m}
$$

where $X_{t-i}$ is the data observation at $i$ time step prior to time $t, i=0,1, \ldots, n ; a_{t-j}$ is the sequence of uncorrelated Gaussian white noise, $j=0,1, \ldots, m ; \phi_{i}$ are autoregressive parameters with the order of $n, i=1,2, \ldots, n$; and $\theta_{j}$ are moving average parameters with the order of $m, j=1,2, \ldots, m$.

The parameters $(\phi, \theta)$ are determined so as to minimize the residuals $J$ based on the leastsquares criterion for the number of $N$ data inputs, $\min J(\phi, \theta)=\sum_{t=1}^{N} a_{t}^{2}$.

The Marquardt nonlinear regression method has been used here to estimate the ARMA model parameters (Pandit and Wu 1983). Two weeks of weather data have been used as historical observations to estimate the ARMA model parameters. One week may be too short a period to contain a sufficient variety of weather condition patterns. A period longer than two weeks could be used, but the results of this study showed no significant improvement in the model accuracy, whereas the computational intensity for estimating the model parameters was increased.

The order of an ARMA model can be identified using two criTerai (Pandit and Wu 1983). The first is the F-criterion, which indicates the improvement in the sum of squares of residuals $\sum a_{t}^{2}$, in going from the lower-order ARMA $(2 n, 2 n-1)$ to the higher-order ARMA $(2 n+2,2 n$ $+1)$. The second criterion ensures accuracy of the ARMA model by checking that the autocorrelations of the residual values of $a_{t}$ are within the $\pm 2 / \sqrt{N}$ band. Under this condition, there is a $95 \%$ confidence level that the expected value of the residual autocorrelations is zero, which ensures that the values of $a_{t}$ are independent of each other. The most appropriate order of the ARMA model for modeling temperature and solar radiation is discussed later in this paper.

The principal difference of ARMA models from the ordinary regression models is that the ARMA models have memory of the disturbance entering the system before the present time. The dynamics or the memory of an ARMA model can be described by two functions. The first is Green's function $G r$, which imposes a stability restriction on the model. The second is the inverse function $I$. The stability and invertibility are checked each time the parameters are estimated for the ARMA model.

\section{ARMAX Model}

To model the influence of solar radiation on the ambient temperature, a multivariable autoregressive moving average vectors system (ARMAV) model has been proposed in this paper. The model formula and associated vectors are

$$
\Phi_{0} Y_{t}=\Phi_{1} Y_{t-1}+\Phi_{2} Y_{t-2}+\cdots+\Phi_{n} Y_{t-n}+e_{t}-\Theta_{1} e_{t-1}-\Theta_{2} e_{t-2}-\cdots-\Theta_{m} e_{t-m}
$$

where

$$
Y_{t-1}=\left[\begin{array}{c}
\bar{T}_{d-i} \\
\bar{G}_{d-i}
\end{array}\right], \quad i=0,1, \ldots, n, \quad e_{t-j}=\left[\begin{array}{c}
a_{t-j} \\
b_{t-j}
\end{array}\right], \quad j=0,1, \ldots, m,
$$




$$
\begin{aligned}
& \Phi_{0}=\left[\begin{array}{cc}
1 & -\varphi_{0} \\
0 & 1
\end{array}\right], \quad \Phi_{i}=\left[\begin{array}{cc}
\phi_{i 1} & \varphi_{i 2} \\
0 & \varphi_{i 1}
\end{array}\right], \quad i=1,2, \ldots, n, \\
& \text { and } \Theta_{j}=\left[\begin{array}{cc}
\theta_{j} & 0 \\
0 & \psi_{j}
\end{array}\right], \quad j=1,2, \ldots, m
\end{aligned}
$$

In this model, a daily time step is applied. $\bar{T}_{d}$ and $\bar{G}_{d}$ are the daily average temperature and the normalized daily total global radiation of day $d . \phi_{i 1}, i=1,2, \ldots, n$ are the autoregressive parameters for the temperature $\bar{T}_{d} . \varphi_{0}$ and $\varphi_{i 2}, i=1,2, \ldots, n$ are the parameters of the exogenous input variable $\bar{G}_{d}$ for modeling the temperature $\bar{T}_{d}$ [see Equation (3)]. $\varphi_{i 1}, i=1,2, \ldots, n$ are the autoregressive parameters for modeling the global radiation $\bar{G}_{d} \cdot \theta_{j}$ and $\psi_{j}, j=1,2, \ldots, m$ are the moving average parameters for the temperature $\bar{T}_{d}$ and the radiation $\bar{G}_{d}$, respectively. Equation (2) is formed under the assumption that the global radiation is an independent time series and that the temperature depends on the global radiation (Yoshida and Terai 1990-1991). The Gaussian noises $a_{t-j}$ and $b_{t-j}$ for the two stochastic processes must also be independent (this is in fact a common assumption for modeling multivariable control systems) (Pandit and Wu 1983).

The parameter estimation of Equation (2) can be accomplished sequentially using an ARMA model for the solar radiation $\bar{G}_{d}$, then the temperature $\bar{T}_{d}$ by an ARMAX model using the past observations of the temperature and the previous and present radiation data as exogenous variables. Equation (3) indicates the ARMAX model for the temperature, derived from Equation (2):

$$
\begin{aligned}
\bar{T}_{d}= & \phi_{11} \bar{T}_{d-1}+\phi_{21} \bar{T}_{d-2}+\cdots+\phi_{n 1} \bar{T}_{d-n}+\varphi_{0} \bar{G}_{d}+\varphi_{12} \bar{G}_{d-1}+\varphi_{22} \bar{G}_{d-2} \\
& +\cdots+\varphi_{n 2} \bar{G}_{d-n}+a_{t}-\theta_{1} a_{t-1}-\cdots-\theta_{m} a_{t-m}
\end{aligned}
$$

The parameter identification for an ARMAX model is the same as that described for the ARMA model.

\section{Deterministic EWMA Model}

An EWMA model has also been used to investigate modeling of both the ambient temperature and global solar radiation. This model is used to remove the deterministic trend of the data. An EWMA model is a special case of the $\operatorname{ARMA}(1,1)$ model with $\phi_{1}=1, \theta_{1}=1-\lambda$. It can also be represented by

$$
\hat{X}=\hat{X}_{t-1}+\lambda\left(X_{t-1}-\hat{X}_{t-1}\right)
$$

Equation (4) shows that the forecast $\hat{X}_{t}$ can be simply computed from $\hat{X}_{t-1}$ and the observation $X_{t-1}$ without needing to store the past observations at time $t$. For the deterministic forecast of temperature and solar radiation required in this paper, a clockwise formulation of the EWMA model has been used:

$$
\hat{D}_{t, d+1}=\hat{D}_{t, d}+\lambda\left(X_{t, d}-\hat{D}_{t, d}\right), \quad t=1,2, \ldots, 24 \text { (hourly) }
$$

where $\hat{D}_{t, d+1}$ is the deterministic forecast for the next day $d+1$ at time $t ; \hat{D}_{t, d}$ is the deterministic forecast for the previous $24 \mathrm{~h}$, day $d$ at time $t$; and $X_{t, d}$ is the temperature or global solar radiation observations for the previous $24 \mathrm{~h}$, day $d$ at time $t$. 
Table 1. Effect of $\lambda$ in EWMA Model

\begin{tabular}{lcccc}
\hline$\lambda$ & RMSE, K & MAE, K & ME, K & MAXAE, K \\
\hline 0.3 & 1.91 & 1.52 & 0.19 & 5.86 \\
0.45 & 1.90 & 1.48 & 0.11 & 6.19 \\
0.6 & 1.92 & 1.48 & 0.07 & 6.62 \\
\hline
\end{tabular}

The only parameter to be determined in the EWMA model for calculating the deterministic element of the data series is the exponential smoothing constant $\lambda$. The smoothing constant $\lambda$ is used to weight the past data. As $\lambda$ increases, greater influence from the more recent observation is given to the model. A large $\lambda$ can result in a rapid response not only to the weather changes, but also to the irregular movements in the time series. However, too small a $\lambda$ may fail to follow the trend of the temperature variations and result in a slow response to changes in the ambient temperature (Abraham and Ledolter 1983). These effects are apparent in Table 1, which illustrates the influence of $\lambda$ on the modeling errors for ambient temperature in September 1994. The lowest value of $\lambda$ results in a prediction that tends to smooth out the extreme errors, and hence a lower MAXAE than for higher values of $\lambda$. However, the low $\lambda$ reduces the ability of the model to follow the general trend of the temperature variations, resulting in a higher ME and MAE. The higher $\lambda$ of 0.6 has an opposite effect, giving the highest MAXAE and RMSE, while the lowest ME and lower MAE. A $\lambda=0.45$ is a good compromise of weighting the historical data, while still providing relatively small modeling errors. Note also that the prediction errors are not sensitive to small changes in $\lambda$. A $\lambda=0.45$ has therefore been adopted in this paper for modeling both the ambient temperature and global solar radiation.

\section{Deterministic Model Based on Sinusoidal Functions}

Modeling the deterministic component of the weather data by sinusoidal functions has also been investigated. Pandit and $\mathrm{Wu}$ (1983) suggested that the sinusoidal functions can be used to model the trend and periodicity of a data series:

$$
\hat{D}_{t, d+1}=\sum_{j=1}^{l} R_{j} \exp \left(r_{j} t\right)+\sum_{j=1}^{n} B_{j} \exp \left(b_{j} t\right)\left[C_{j} \sin (j \omega t)+\sqrt{1-C_{j}^{2}} \cos (j \omega t)\right]
$$

where $B_{j}, b_{j}, C_{j}, r_{j}$, and $R_{j}$ are parameters to be estimated. For the temperature data, $\omega=2 \pi / 24$. For the radiation data, because the radiation at night is deterministically zero, in order to reduce prediction errors, $\omega=2 \pi / 17$ is applied. The time of sunrise and sunset is site dependent, and a different cycle or $\omega$ value for modeling solar radiation using sinusoidal functions may be used for other climate types. The raw radiation data are used here and Equation (6) is applied to find the best fit of the data using sinusoidal functions. Solar radiation data can also be normalized before applying Equation (6). It is anticipated that using daily total radiation to normalize the hourly radiation data would not eliminate the site dependence of the data. Other methods, such as normalizing the radiation data using theoretical calculations of hourly radiation for a particular site, have not been investigated in this paper.

The Marquardt nonlinear estimation routine has been used to calculate $R_{j}$ and $r_{j}(j=1,2, \ldots, l)$, and $B_{j}, b_{j}$, and $C_{j}(j=1,2, \ldots, n)$. It was found that the exponential growth trend in the temperature and radiation was very small and could be neglected, $l=1, r_{1}=0$, and the third order $(n=3)$ of periodic trend is sufficient for modeling the temperature data. Table 2 gives an example of the values for $B_{j}, b_{j}$, and $C_{j}(j=1,2,3)$ and $R_{1}$ of the third-order model using the temperature observations in March of the CIBSE example weather year. The parameters of the model can also be updated daily. 
Table 2. Example of Parameters Fitted for Sinusoidal Functions

\begin{tabular}{ccccc}
\hline Number Order & B & b & c & R \\
\hline First & -3.56 & -0.01 & 0.86 & 8.78 \\
Second & 8.56 & -0.34 & 0.99 & - \\
Third & -4.61 & -0.33 & 1.00 & - \\
\hline
\end{tabular}

\section{TEMPERATURE PREDICTION}

Three methods of using ARMA models have been investigated here. The first approach, the stochastic method (SM), is to model the nonstationary temperature time series, using the raw temperature data to estimate the parameters of the ARMA model. In the second approach, the deterministic-stochastic method (DSM), the stationary stochastic time series is modeled by subtracting the deterministic component out of the raw temperature data so that the stochastic element can be calculated by a reduced order of the ARMA model. The deterministic component represents the periodicity and trend of the hourly ambient temperature. The third method expands on the DSM to include the effect of global solar radiation on the temperature prediction, and has therefore been called the expanded deterministic-stochastic method (EDSM).

\section{Stochastic Method}

In SM, the next day's temperature profile is predicted using only the ARMA model of Equation (1). Using the ambient temperature data for July of the CIBSE example year, the results indicated that, to meet the F-criterion, a high order of $\operatorname{ARMA}(26,25)$ was required. Because the ambient temperature changes in a diurnal cycle of $24 \mathrm{~h}$ randomly hour by hour, the hourly sampled data may be represented by an ARMA model of an approximate order of $(n \times 24)$, with the exact value of periodicity of the model varying marginally with different periods of temperature sampled [in this case, $\operatorname{ARMA}(26,25)]$. If the moving average parameters are removed and an AR model is used, a higher number of parameters of $\operatorname{AR}(2 n)$ is required. In this instance an $\operatorname{AR}(36)$ results in a similar value of $\sum a_{t}^{2}$ to an $\operatorname{ARMA}(26,25)$. It can be concluded that, although the ARMA model is simple in structure, a high order of the parameters is required to model the diurnal ambient temperature series.

\section{Deterministic-Stochastic Method}

It is likely that the order of an ARMA model can be reduced by separately modeling the deterministic periodicity of the ambient temperature time series. The procedure for establishing the combined model is in three steps:

1. Predict the deterministic part $\hat{D}_{t, d+1}$ of the time series using either the EWMA model, Equation (5), or the sinusoidal function, Equation (6).

2. Calculate the stochastic part of the time series $S_{t}$ from the errors in the deterministic predictions for the previous $N$ data samples, $S_{t}=T_{t}-\hat{D}_{t}, t=1,2, \ldots, N$ ( $N$ being equal to 14 days of hourly sampled data in this case).

3. Predict the stochastic element of the time series $\hat{S}_{t}$, using an ARMA model, Equation (1).

The temperature forecasts $\hat{T}_{t, d+1}(t=1,2, \ldots, 24)$ are then given by combining the predictions for the deterministic and stochastic elements:

$$
\hat{T}_{t, d+1}=\hat{D}_{t, d+1}+\hat{S}_{t, d+1} \quad(t=1,2, \ldots, 24)
$$

The results indicate that an $\mathrm{AR}(4)$ is adequate to model the stochastic element of the time series. 

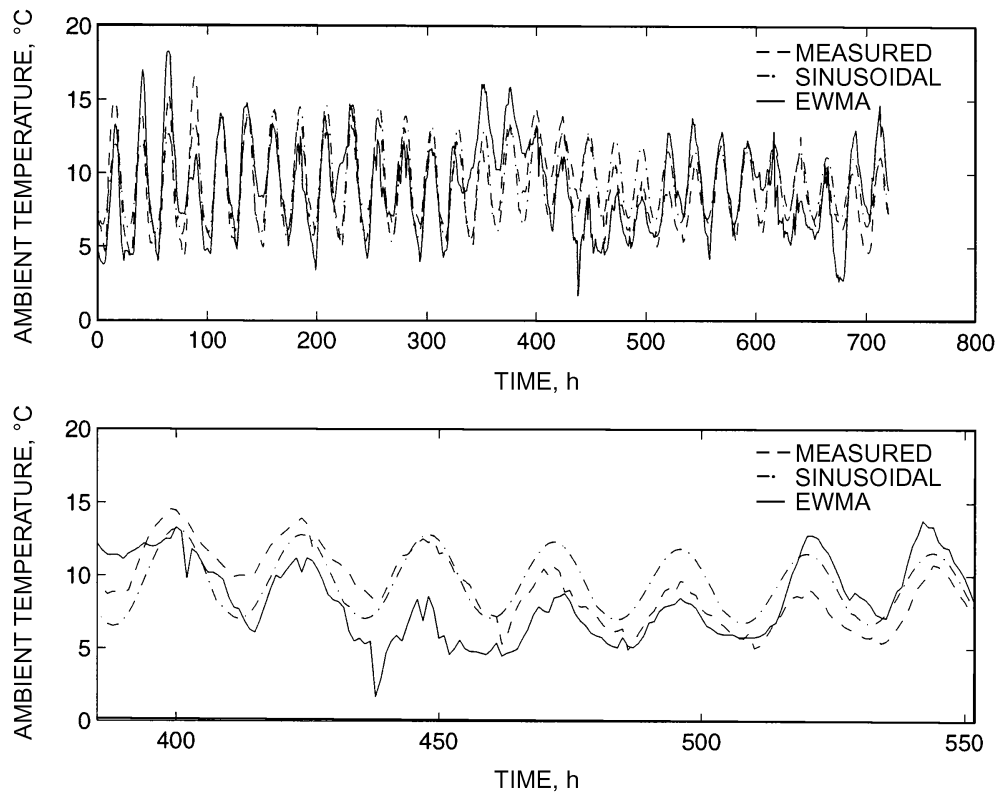

Figure 3. Comparison Between Sinusoidal Functions and EWMA in Modeling Deterministic Element of Temperature Profile

Table 3. Comparison of Sinusoidal Functions and EWMA Model

\begin{tabular}{lcccc}
\hline Methods & RMSE, K & MAE, K & ME, K & MAXAE, K \\
\hline Sinusoidal & 2.35 & 1.86 & 0.31 & 6.73 \\
EWMA & 2.10 & 1.53 & 0.00 & 7.13 \\
\hline
\end{tabular}

The performance of the EWMA model and sinusoidal functions in modeling the deterministic element of the time series are compared in Table 3 for April of the CIBSE weather year. The relative performance is also illustrated in Figure 3 (the lower graph illustrates the data from April 17 to 23). It can be observed that the sinusoidal functions do not adapt effectively to the varying trend of the temperature, even though the parameters of the functions are updated each day. This results in the sinusoidal function giving higher RMSE and MAE, but a smaller MAXAE than the EWMA model. The EWMA model predicts the temperature series without bias and has smaller errors than the sinusoidal function, except for the MAXAE, this being $0.4 \mathrm{~K}$ higher than the sinusoidal method. In effect, using sinusoidal functions results in a similar performance to the EWMA model having a lower $\lambda$. It was also found that the shape of diurnal temperature variations affects the performance of the sinusoidal functions. For instance, in July, when the amplitude of diurnal temperature is large and the cycle of the diurnal variation is clearly defined, the sinusoidal functions give a more accurate prediction than for April or the winter months.

In comparison to the EWMA model, the sinusoidal function model has the disadvantage of using a computationally intensive nonlinear parameter estimation method. Therefore, considering the relative accuracy of the two methods, the combined deterministic-stochastic model adopted in the reminder of this study is an EWMA (with $\lambda=0.45$ ) for the deterministic component, combined with an $\mathrm{AR}(4)$ for the stochastic component. 
The influence of the daily total global radiation on the ambient temperature prediction has been examined by expanding the deterministic-stochastic model to include the total daily global radiation as an exogenous variable. The expanded model is formed in two parts:

1. Daily average temperature, $\widehat{T}_{d+1}$ is predicted using the ARMAX model [Equation (3)], with the total global radiation $\bar{G}_{d+1}$ as the exogenous variable;

2. Hourly sampled temperature data are then subtracted from the daily average temperature and the remaining time series modeled by the DSM [Equations (5) and (1), with $\lambda=0.45$ EWMA for the deterministic part and a $\operatorname{AR}(2)$ for the stochastic part].

The temperature forecasts $\hat{T}_{t, d+1}(t=1,2, \ldots, 24)$ are then given by combining the predictions for the daily average temperature with the deterministic and stochastic predictions for the remaining time series:

$$
\hat{T}_{t, d+1}=\widehat{T}_{d+1}+\hat{D}_{t, d+1}+\hat{S}_{t, d+1} \quad(t=1,2, \ldots, 24)
$$

The daily total global radiation is normalized $\bar{G}_{d}$, where $\bar{G}_{d}=G_{d} / H_{o} . G_{d}$ is the daily total global radiation received on the horizontal surfaces and $H_{o}$ is extraterrestrial daily insolation on the horizontal surfaces (Liu and Jordan 1960). The normalization has the effect of exaggerating the local climatic effects, such as cloud cover, on the total global radiation received at the earth's surface. $H_{O}$ is calculated by

$$
H_{o}=\frac{24}{\pi} r I_{s c}\left(\cos L \cos \gamma \sin \omega_{s}+\omega_{s} \sin L \sin \gamma\right)
$$

where $L, \gamma$, and $\omega_{s}$ are latitude, solar declination, and sunset hour angle respectively (radians). $r$ is ratio of solar radiation intensity (at normal incidence to the outside of the atmosphere of the earth) to the solar constant, (dimensionless); $r$ depends on the distance between the earth and the sun. $I_{s c}$ is the solar constant. $H_{o}$ has little variation within a given month (Liu and Jordan 1960).

One month of daily average sampled temperature data has been used to estimate the parameters of the ARMAX model for the daily average temperature [Equation (3)]. This gave greater accuracy than the two weeks of weather data used for the DSM, but without imposing an excessive computational penalty. An AR(2) ARMA model was identified as providing suitable accuracy for predicting the daily global radiation $\bar{G}_{d}$. An ARMAX model of order ARX(2) also proved to have sufficient accuracy to model the daily average temperature.

Three investigations of the accuracy of the temperature prediction models have been carried out:

- Comparison of temperature prediction from stochastic and deterministic-stochastic models

- Investigation into the effect of global solar radiation on daily average temperature prediction

- Comparison of expanded-deterministic-stochastic model prediction with the deterministicstochastic model prediction

Four conventional error measures have been used to compare the prediction from the SM, [Equation (1)], with the DSM [Equation (7)]. Table 4 gives the comparison in July of the CIBSE year between the SM and the DSM. The DSM gives smaller RMSE, MAE, and MAXAE, while having a small positive bias ME. A comparison has also been conducted on the temperature prediction for all other months of the CIBSE year and 1994, with a similar result. The DSM is simpler, less computationally intensive, and generally results in smaller prediction errors than the SM. 
Table 4. Performance Comparison of Stochastic and Deterministic-Stochastic Methods

\begin{tabular}{lcccc}
\hline Methods & RMSE, K & MAE, K & ME, K & MAXAE, K \\
\hline SM & 2.07 & 1.50 & -0.01 & 8.04 \\
DSM & 1.88 & 1.41 & 0.13 & 6.62 \\
\hline
\end{tabular}

Table 5. Prediction Errors for CIBSE Year and 1994 from Combined Deterministic-Stochastic Method

\begin{tabular}{lcccc}
\hline Periods & RMSE, K & MAE, K & ME, K & MAXAE, K \\
\hline CIBSE year & 2.14 & 1.58 & 0.01 & 10.40 \\
1994 & 2.46 & 1.81 & -0.02 & 14.25 \\
\hline
\end{tabular}

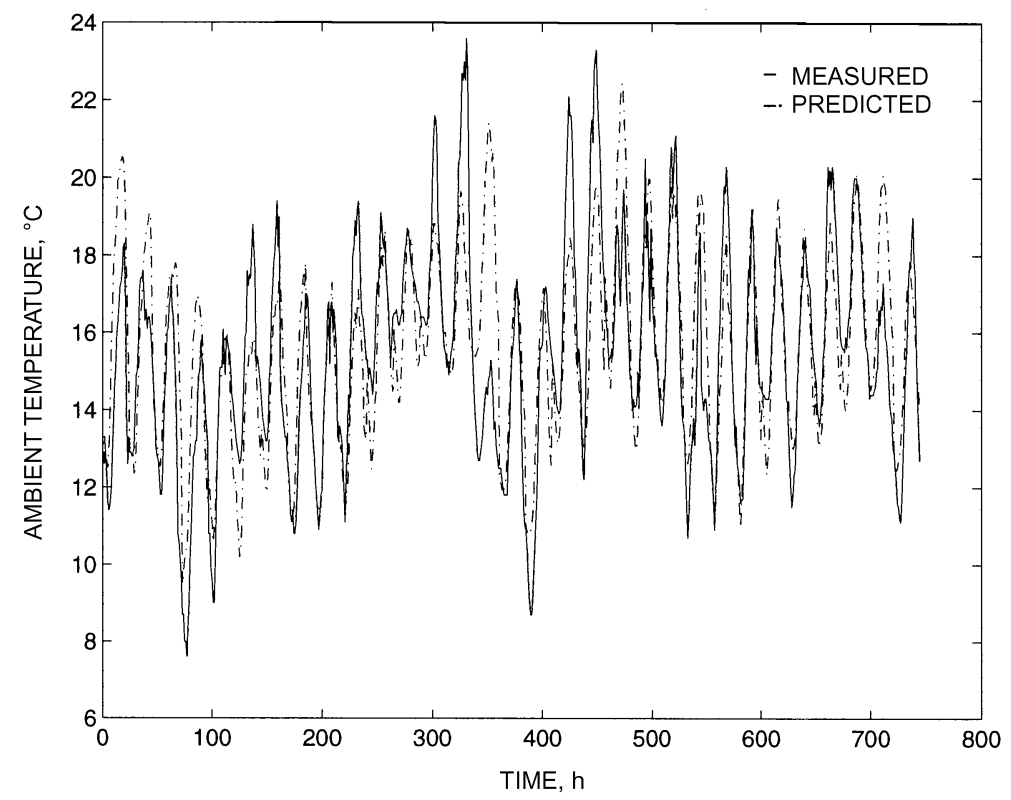

Figure 4. Ambient Temperature Prediction Profile for July of CIBSE Year

The prediction errors from the DSM for the CIBSE year and 1994 are given in Table 5. Although the MAXAE are large because of the random variations in temperature, the average RMSE and MAE are acceptable for the two sets of weather data and have little or no bias ME. The small average errors indicate that this method can follow the general trend of ambient temperature variations.

Figure 4 shows the ambient temperature prediction for July of the CIBSE year and Figure 5 for September of 1994 using the DSM. The model is able to follow the pattern of temperature change, although when a sudden change in weather occurs, the model can take one or two days to realign with the new pattern. For instance, on July 15 (Figure 6), there was a sudden drop in temperature of approximately $8.3 \mathrm{~K}$, which inevitably leads to larger prediction errors. However, by the next day the model realigns with the new pattern.

To justify the influence of the solar radiation on the daily average temperature, $\bar{T}_{d}$ has been modeled by two methods: (1) a standard AR(2), modeling the temperature independently and ignoring the correlation to the daily total radiation; and (2) an ARX(2) to include the effect of solar radiation [Equation (3)]. The accuracy of the two methods is compared in Table 6 for September 1994. 
Table 6. Influence of Solar Radiation on Prediction of Daily Average Temperature

\begin{tabular}{lcccc}
\hline Methods & RMSE, K & MAE, K & ME, K & MAXAE, K \\
\hline Independent, AR(2) & 1.38 & 1.11 & 0.66 & 3.01 \\
Correlated, ARX(2) & 1.47 & 1.15 & 0.76 & 3.31 \\
\hline
\end{tabular}

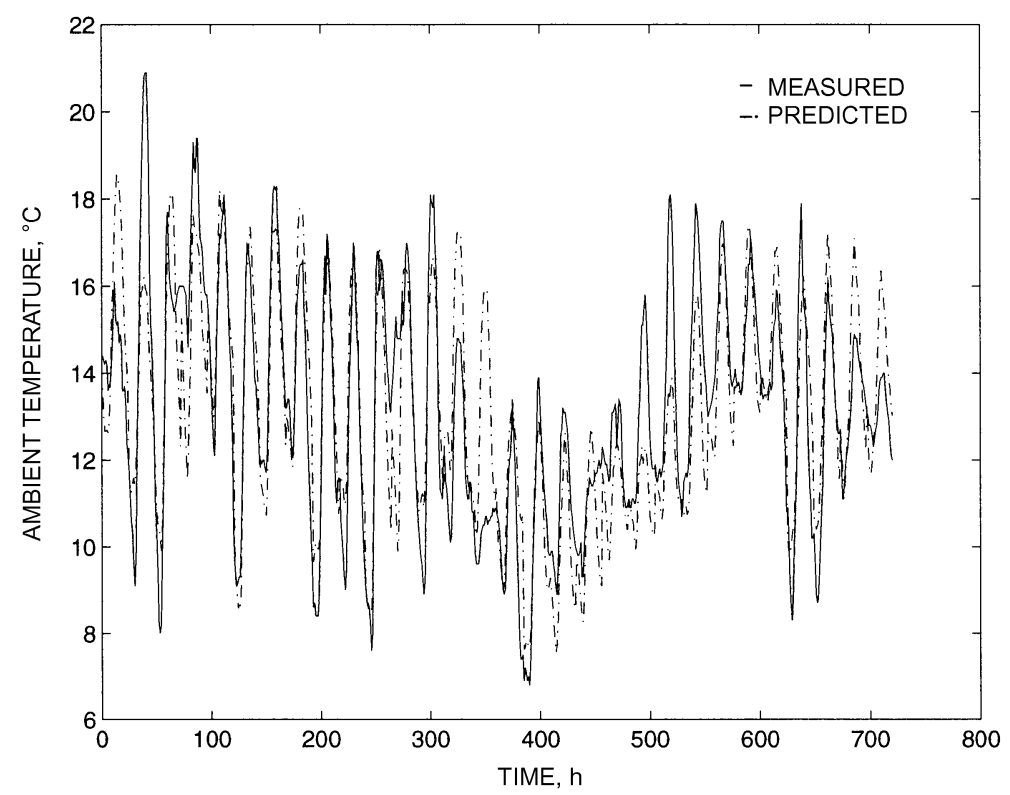

Figure 5. Ambient Temperature Prediction Profile for September 1994

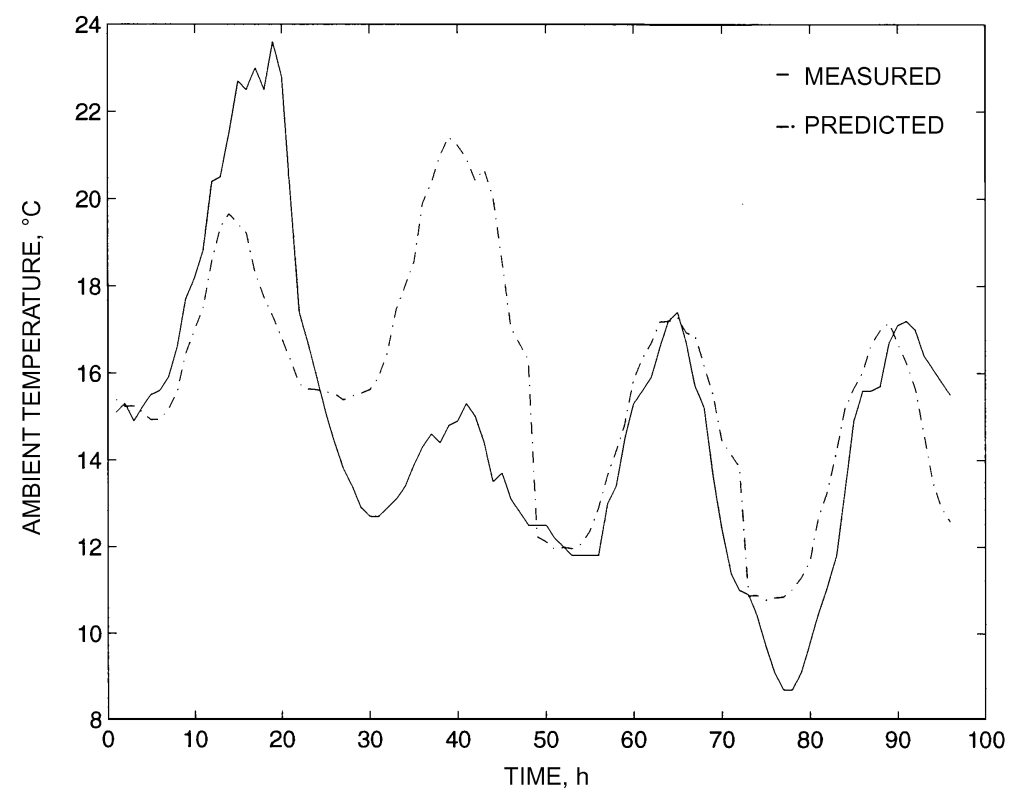

Figure 6. Ambient Temperature Prediction Profile for July 14 to 17 of CIBSE Year 
All the errors from the independent model are smaller for the September data. Because of the highly random behavior of the global radiation, the correlation between the daily average temperature and the total radiation during each month is uncertain. The correlation for September 1994 is approximately equal to the average value of the monthly calculated correlations, $\rho_{x y}=0.2$. This study indicates that the error differences between the two methods did not change significantly with the correlation for other months, including those with a higher correlation between global radiation and ambient temperature. Therefore, it can be concluded that the daily average temperature can be modeled with sufficient accuracy by its past observations, without reference to global radiation.

The ARX-EWMA-AR method and the EWMA-AR methods were compared using the predictions for the daily average ambient temperature $\bar{T}$ and the amplitude of the temperature variations $\Delta T$. In the ARX-EWMA-AR method, the ambient temperature is modeled in two parts: the daily average temperature is calculated by an $\operatorname{AR}(2)$, and the amplitude of hourly temperature variations by the same procedure as for the DSM. In the EWMA-AR method, the daily average temperature $\bar{T}$ and amplitude $\Delta T$ are not modeled separately, so they have been calculated from the predicted temperature $T$ for comparison purposes.

The results from the expanded method are displayed in Table 7, where the forecast errors of daily average temperature $\bar{T}$, amplitude of the temperature variations $\Delta T$, and ambient temperature $T(T=\bar{T}+\Delta T)$ are compared with those from the combined method.

In Table 7, the subscripts 7 and 9 have been added to denote the errors for July of the CIBSE year and September of 1994. Most errors from the combined method are smaller than those from the expanded method. Although the prediction error MAXAE of the daily average temperature for July is $0.4 \mathrm{~K}$ larger and has more bias (ME) from the combined method, the average errors RMSE and MAE are both smaller. It also better predicts the amplitude of the temperature variations. The errors for September indicate that all the errors from the combined method are smaller than those from the expanded method. The comparison of the temperature prediction for other periods illustrates that the two methods result in a similar range of errors; however, the combined method is slightly more accurate and much simpler in structure and operation.

From the investigation on the performance of the pure SM, the combined DSM, and the expanded combined method (Tables 4 and 7), it is justified that the combined DSM is competent in accuracy and simplicity for short-term forecasting of temperature, and it has therefore been adopted to model and forecast the ambient temperature for the predictive control of thermal storage systems.

In the three prediction methods, the ambient temperature is predicted for the next $24 \mathrm{~h}$ only once at the end of every day, the information being used to calculate the optimum control strategy for the next $24 \mathrm{~h}$. It is, however, possible to improve the temperature prediction for the future hours of the day by the use of observations of the past hours.

Table 7. Performance Comparison of Two Combined Methods

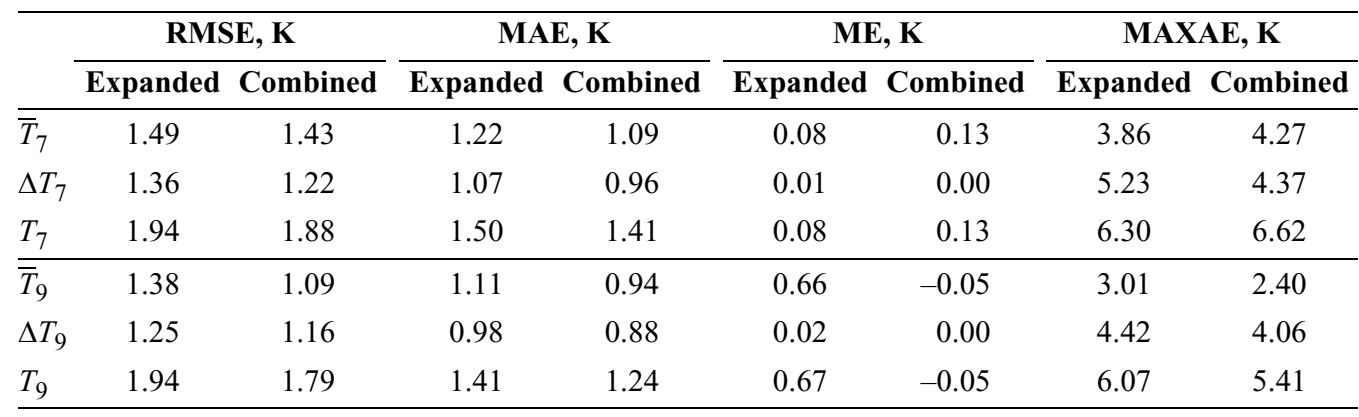


For forecasting a longer lead time using an ARMA model, the temperature prediction error is inevitably larger later in the day than in the early morning. With the new temperature observations of the following day being available, the parameters of the ARMA model and the forecast can be updated to improve the accuracy by re-estimating the parameters of the ARMA model or simply by using Green's function (Pandit and Wu 1983).

Let $\hat{X}_{t}(i)$ be a forecast for a lead time $i$ at time $t$, and $X_{t+i}$ be the observation at time $t+i$. In this weather prediction model, the forecast for the lead time from $i=1$ to $24 \mathrm{~h}$ of the next day is required at the end of every day. The updating can be achieved by Green's function,

$$
\hat{X}_{t+1}(i)=\hat{X}_{t+1}(i+1)+G r_{i}\left[X_{t+1}-\hat{X}_{t}(i)\right]
$$

This equation states that at time $t+1$, the updated forecast $\hat{X}_{t+i}$ of the observation $X_{t+1+i}$ is obtained from the old forecast $\hat{X}_{t}(i+1)$ at time $t$ simply by adding $G r_{i}$ times the new shock $\left[X_{t+1}-\hat{X}_{t}(i)\right]$, which becomes known once $X_{t+1}$ is known.

The performance of adapting to the new observations by re-estimating the ARMA model parameters has been compared with using Green's function. It was found that the two methods have similar performance, although Green's function gives a slightly quicker response to the new temperature observations.

Updating the temperature forecast by using Green's function is simple and efficient, but only in improving the forecast accuracy for a short lead time. Because of the randomness of temperature variations, the updated profile of temperature prediction during the daytime may be less accurate than one not updated. For instance, as illustrated in Figure 7, if the updating is undertaken at 2:00 A.M., the forecast for 3:00 A.M. and 4:00 A.M. can be improved. However, because the observed temperatures at 2:00 A.M. and 1:00 A.M. are lower than the original predictions, updating the prediction at 2:00 A.M. lowered the predicted temperatures for the rest of the day, which resulted in higher prediction errors during the occupied period (Figure 7). Furthermore, the temperature prediction errors are small during the night because the lead time is short. It can therefore be concluded that updating the prediction profile according to the observations during the night hours is not efficient in improving the forecasting of the daytime temperatures.

Updating the prediction at the beginning of the occupancy period can improve the temperature prediction for the occupancy period (Figure 7, updating at 8:00 A.M.). This may be useful for applications such as hourly predictive control using a local loop controller (Holst et al. 1987). However, for predictive control of thermal storage, this procedure loses its significance because the time for planning to use the potential of the plant night operation to offset the daytime thermal loads has passed. Therefore, the control strategy optimization is only conducted once, at the beginning of the day, and the temperature updating can be used to improve hourly plant operation during the daytime.

The temperature prediction can also be corrected by the forecast daily maximum and minimum temperatures obtained from a local weather station. Supposing that $\hat{T}_{\text {max }}$ and $\hat{T}_{\text {min }}$ and the variation $\hat{\delta}\left(\hat{\delta}=\hat{T}_{\text {max }}-\hat{T}_{\text {min }}\right)$ are calculated from the predicted temperature profile, and $T_{\max }, T_{\min }$, and the variation $\delta\left(\delta=T_{\max }-T_{\min }\right)$ are provided by the forecast from the local weather station, the corrected $\tilde{T}_{t}$ at time $t$ can be calculated by

$$
\tilde{T}_{t}=\frac{\delta}{\hat{\delta}}\left(\hat{T}_{t}-\hat{T}_{\text {min }}\right)+T_{\text {min }}
$$

If the forecast of the daily maximum and minimum temperatures from the weather station is accurate, the corrected profile significantly improves the temperature prediction during the day- 


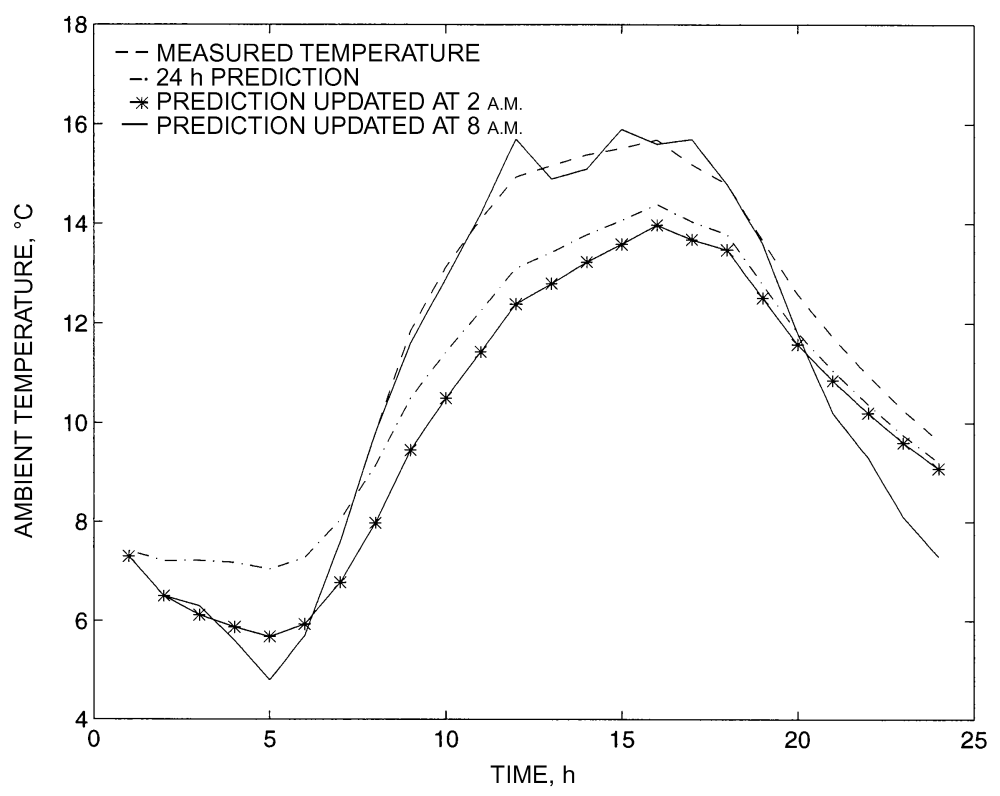

Figure 7. Updating Temperature Profile for May 9, 1994

time. However, results show that this correction procedure, in effect, enlarges or contracts the predicted temperature profile according to the input of daily maximum and minimum temperatures. Because the prediction errors are usually small during the night and early morning (the lead time is short), the correction is likely to increase the errors for this period by enlarging or contracting the profile.

The updating is effective in improving temperature predictions for the short lead time and the correction for the daytime profile, so it is possible to combine the use of temperature observations to update the forecast of adjacent future hours and the correction of the temperature profile predictions for the daytime.

\section{RADIATION PREDICTION}

Like ambient temperature, solar radiation is a nonstationary stochastic data series. The hourly sampled radiation data (global, diffuse and direct) exhibit even more random behavior than the temperature data. The global, diffuse, and direct radiation are all modeled, but only the global radiation is taken as the example here to illustrate the prediction model.

In order to apply a stochastic model to the radiation data series, the series has been reconstructed to a $17 \mathrm{~h}$ periodicity instead of $24 \mathrm{~h}$, (from 5:00 A.M. to 9:00 P.M. when there is noticeable incidence of solar radiation on the earth; shorter hours of periodicity can be used for the winter period). Although the periodicity of solar radiation depends on the site and climate type, it will be seen from the following investigation that a solely deterministic model is required for predicting solar radiation; a stochastic model could not sufficiently reduce the prediction errors. Therefore, the recommended prediction model for solar radiation does not require reconstructing a radiation data series to a specific cycle.

The solar radiation can be modeled independently. Similar to the temperature prediction models, the ARMA model and two deterministic models have been used in this paper to investigate the radiation prediction. From the results of the temperature model comparisons, the combined 
Table 8. Performance Comparison

\begin{tabular}{|c|c|c|c|c|}
\hline Methods & RMSE, W/m ${ }^{2}$ & MAE, W/m 2 & ME, W/m $\mathbf{m}^{2}$ & MAXAE, W/m ${ }^{2}$ \\
\hline$\overline{\text { EWMA }}$ & 116.08 & 68.69 & 3.50 & 473.38 \\
\hline Sinusoidal & 155.00 & 111.37 & 29.14 & 554.23 \\
\hline
\end{tabular}

DSM has been shown to accurately model the nonstationary temperature data series. This method can also be used to predict the solar radiation, because the ambient temperature and solar radiation have similar deterministic and stochastic properties.

In the combined radiation method, the radiation data are divided into two parts, deterministic and stochastic. The stochastic part is modeled by the ARMA time-series technique, the deterministic part by either the EWMA model or the sinusoidal functions.

As in the temperature prediction, EWMA model is applied to the global radiation data, with $\lambda=0.45$. For the sinusoidal function method, Equation (6) is used. Table 8 compares the performance between the EWMA and the sinusoidal function method for July of the CIBSE year. The prediction errors from the EWMA model are all much smaller, with nearly no bias (ME) and smaller average errors (RMSE and MAE) and MAXAE. The EWMA model has, therefore, been used for modeling the deterministic part of the solar radiation. The EWMA model can also eliminate the need for constructing the solar radiation data series to a specific periodicity according to a site.

The stochastic part, comprised of the errors in the deterministic prediction of the radiation data, still appears to be nonstationary, with a much higher mean and standard deviation in the middle of the day than during the morning and evening hours. The periodicity of the stochastic part is unstable because of the dramatic variations in amplitude (hourly variations of the global radiation indicate a frequent and sharp increase or decrease in some consecutive hours of one day). It has been found that the ARMA model fails to model the stochastic part: the solar radiation varies randomly with such high frequency and large amplitude (Figure 8) that the prediction from the ARMA model appears to be a filtered response to a high-frequency disturbance, which is in fact the prediction error from the deterministic model. By including the stochastic part, the error in modeling the global radiation is not significantly reduced from that using the deterministic prediction only. The purely deterministic, clockwise EWMA model has, therefore, been adopted here to model the solar radiation. This method also removes the need for reconstructing solar radiation data series to a specific periodicity.

Figure 9 gives an example of a 1 week prediction of the global radiation from September 17 to 23,1994 . The EWMA model gives reasonable accuracy in forecasting the highly stochastic solar radiation.

Hourly observations of solar radiation are not always measured by building control systems, but the daily total global radiation can be obtained from a local weather station or by prediction. In this case, an empirical ratio can be used to transfer the daily sampled data to hourly values (Liu and Jordan 1960). Duffie and Beckman (1974) demonstrated the use of the ratio of hourly average radiation to daily average radiation to estimate the hourly value from daily data. The solar altitude was also used to calculate the radiation in each hour received on the horizontal surfaces (Yoshida and Terai 1992). If either the diffuse or direct radiation is not available, the interrelationship between the different radiation data can be calculated (Liu and Jordan 1960). This approach has not been investigated further here.

\section{CONCLUSIONS}

Three temperature prediction methods have been investigated: the pure SM, the combined DSM, and the expanded method for short-term temperature forecast. It has been found that the 


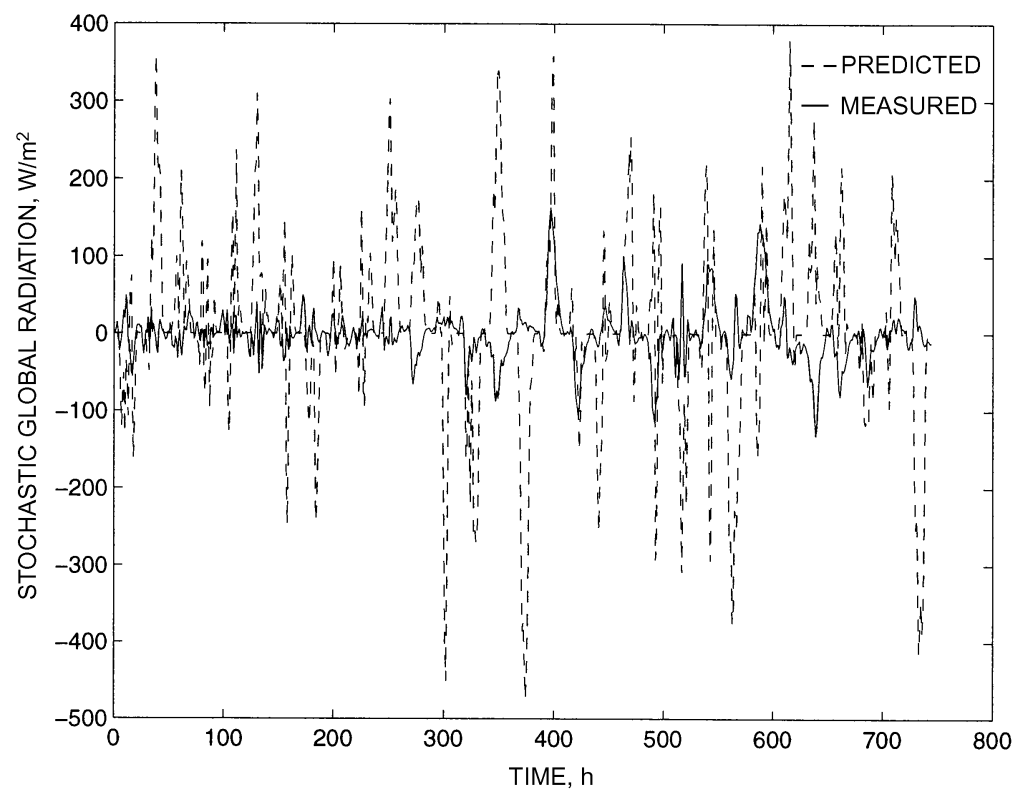

Figure 8. Modeling of Stochastic Element of Global Radiation

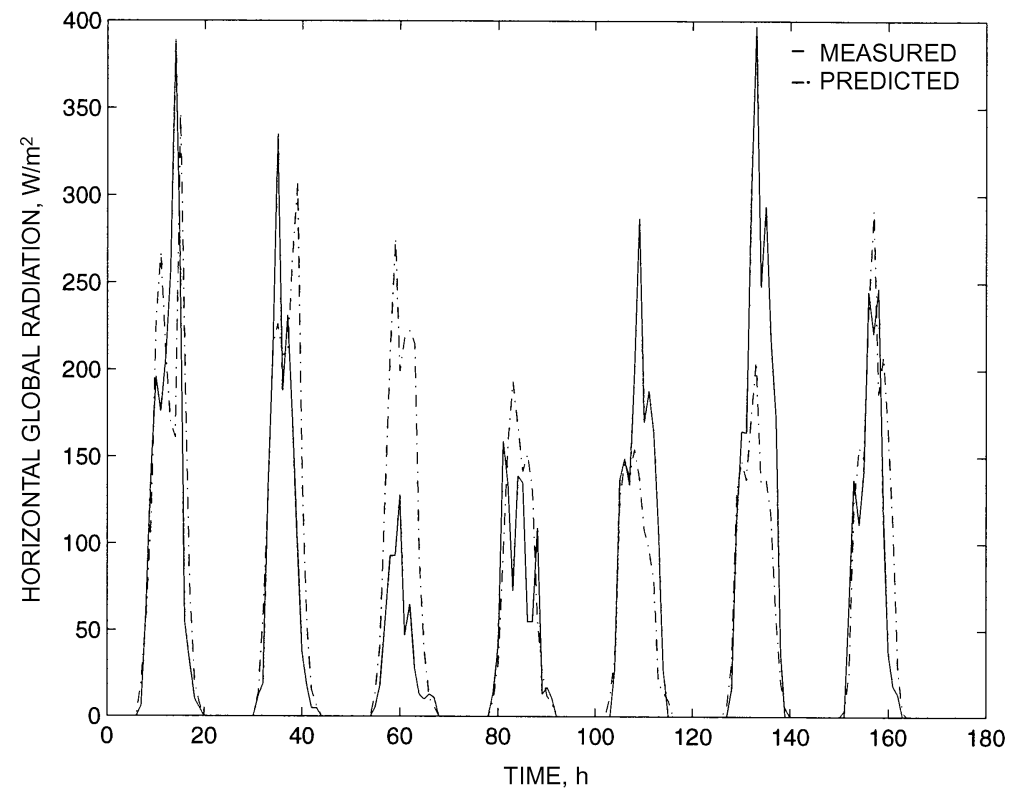

Figure 9. Prediction Profile of Global Radiation from September 17 to 23, 1994

combined DSM is simpler and most likely provides smaller prediction errors. The investigation of the influence of correlation between the solar radiation and the temperature has indicated that it is not necessary to consider the influence of the daily total solar radiation on the daily average temperature for the short-term temperature forecast. Further, the daily average temperature does not need to be modeled separately. The combined DSM can provide an acceptable accuracy in 
predicting the daily average, amplitude of temperature variations, and ambient temperature. In addition, the investigation of updating the weather prediction within a day has shown that it is not effective in reducing the forecasting errors of the daytime temperature and improving the optimum scheduling of plant operation for the next day. To improve accuracy of the temperature profile forecast, it is possible to combine updating the prediction by the temperature observations and correction using the daily maximum and minimum temperature forecast available from a local weather station.

In conclusion, the adaptive algorithm for temperature prediction used in this paper consists of a deterministic part and a stochastic part, which model the deterministic trend and stochastic variations of the ambient temperature. An EWMA model has been used to account for the deterministic part, and an AR(4) model for the stochastic part of the temperature. Using this method to model the two sets of weather data, the mean absolute error in predicting the ambient temperature over an entire year is less than $1.8 \mathrm{~K}$ and the root mean square error less than $2.5 \mathrm{~K}$, with no bias. For predicting solar radiation, the deterministic EWMA model has been used. The algorithm is simple and efficient for use in off-line parameter estimation at the end of every day.

Although the effectiveness of the method has not been investigated for other climates, it is anticipated that because the method has been examined for both stable (such as winter and summer months in the U.K.) and more random conditions (like transitional seasons in the U.K.), it will offer good performance over the range of climates experienced in other countries.

\section{NOMENCLATURE}

a uncorrelated Gaussian white noise

$b \quad$ uncorrelated Gaussian white noise; sinusoidal function coefficient

$B \quad$ sinusoidal function coefficient

$C$ sinusoidal function coefficient

$\hat{D}$ deterministic forecast

$e \quad$ vector of uncorrelated Gaussian white noise

$G$ daily total global radiation

$\bar{G}$ normalized daily total global radiation

$\mathrm{Gr}$ Green's function

$H_{o}$ extraterrestrial daily insolation on the horizontal surfaces

I inverse function

$I_{s c} \quad$ solar constant

$L \quad$ latitude of a particular site, radians

$N \quad$ number of data samples

$r \quad$ ratio of solar radiation intensity to the solar constant; and sinusoidal function coefficient

$R \quad$ sinusoidal function coefficient

$S_{t} \quad$ stochastic part of the data series

$\hat{S}_{t} \quad$ forecast of stochastic element of the data series

$T$ temperature

$\bar{T} \quad$ daily average temperature

$\hat{T} \quad$ temperature forecast

$\overparen{T}$ daily average temperature forecast

$\tilde{T} \quad$ corrected temperature prediction

$\Delta T \quad$ amplitude of temperature variation

$X$ data observation

$\hat{X} \quad$ forecast of data observation

$Y \quad$ vector of daily average temperature $\bar{T}$ and normalized daily total radiation $\bar{G}$

\section{Greek}

$\gamma \quad$ solar declination, radians

$\delta \quad$ difference in daily maximum and minimum temperature

$\hat{\delta} \quad$ predicted difference in daily maximum and minimum temperature

$\theta \quad$ moving average parameters

$\Theta \quad$ matrix of moving average parameters

$\lambda$ exponential smoothing constant

$\rho_{x y} \quad$ correlation coefficient between $x$ and $y$

$\phi \quad$ autoregressive parameters

$\phi_{i 1} \quad$ autoregressive parameters, for the daily average temperature $\bar{T}$

$\varphi_{0} \quad$ parameter of exogenous input variable $\bar{G}$

$\varphi_{i 1} \quad$ autoregressive parameters for the normalized daily total global radiation $\bar{G}$

$\varphi_{i 2} \quad$ parameters of the normalized daily total global radiation $\bar{G}$ as an exogenous input variable

$\Phi \quad$ matrix of autoregressive parameters

$\Phi_{0} \quad$ matrix of parameters for daily average temperature $\bar{T}$ and normalized daily total radiation $\bar{G}$

$\psi \quad$ moving average parameters for the normalized daily total global radiation $\bar{G}$

$\omega \quad$ cycle of sinusoidal functions

$\omega_{s} \quad$ sunset hour angle, radians 


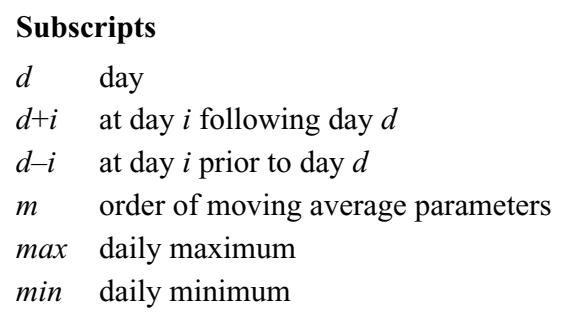

$n \quad$ order of autoregressive parameters, and number of sinusoidal function coefficients

$t \quad$ time

$t+i \quad$ at $i$ time steps following time $t$

$t-i \quad$ at $i$ time steps prior to time $t$

$t-j \quad$ at $j$ time steps prior to time $t$

$t, d \quad$ at time $t$ and day $d$

$t, d+1$ at time $t$ and for the next day $d+1$

\section{REFERENCES}

Abraham, B. and J. Ledolter. 1983. Statistical Methods for Forecasting. New York: John Wiley \& Sons.

Athienitis, A.K. 1988. A Predictive Control Algorithm for Massive Buildings. ASHRAE Transactions 94(2):1050-1067.

Chen, T.Y. and A.K. Athienitis. 1996. Ambient Temperature and Solar Radiation Prediction for Predictive Control of HVAC Systems and a Methodology for Optimal Building Heating Dynamic Operation. ASHRAE Transactions 102(1):26-36.

Duffie, J.A. and W.A. Beckman. 1974. Solar Energy Thermal Processes. New York: John Wiley \& Sons.

Ferrano, F.J. and K.V. Wong. 1990. Prediction of Thermal Storage Loads Using a Neural Network. ASHRAE Transactions 96(2):723-726.

Forrester, J.R. and W.J. Wepfer. 1984. Formulation of a Load Prediction Algorithm for a Large Commercial Building. ASHRAE Transactions 90(2B):536-551.

Holst, J., H. Madsen, and P. Thyregod. 1987. A Method for Using Hourly Predictions of Weather Observations in Optimal Control of Heat Supply to Buildings. Proceedings of 3rd International Congress on Building Energy Management, ICBEM, pp. 380-389.

Jiang, Y. and T. Hong. 1993. Stochastic Analysis of Building Thermal Process. Building and Environment 28(4):509-518.

Kawashima, M., C.E. Dorgan, and J.W. Mitchell. 1995. Hourly Thermal Load Prediction for the Next 24 Hours by ARIMA, EWMA, LR, and an Artificial Neural Network. ASHRAE Transactions 101(1): 186-200.

Keeney, K.R. and J.E. Braun. 1997. Application of Building Precooling to Reduce Peak Cooling Requirements. ASHRAE Transactions 103(1):463-469.

Liu, B.Y.H. and R.C. Jordan. 1960. The Interrelationship and Characteristic Distribution of Direct, Diffuse and Total Solar Radiation. Solar Energy 4:1-19.

MacArthur, J.W., A. Mathur, and J. Zhao. 1989. On-Line Recursive Estimation for Load Profile Prediction. ASHRAE Transactions 95(1):621-628.

Pandit, S.M. and S.M. Wu. 1983. Time Series and System Analysis with Applications. New York: John Wiley \& Sons.

Ren, M.J. 1997. Optimal Predictive Control of Thermal Storage in Hollow Core Ventilated Slab Systems, $\mathrm{Ph} . D$. dissertation. Loughborough University, Loughborough, Leicestershire, U.K.

Ren, M.J. and J.A. Wright. 1997. Predictive Optimal Control of Fabric Thermal Storage Systems. Proceedings of Building Simulation '97. Prague, Czech Republic, Volume II, pp. 71-79.

Rupanagunta, P., M.L. Baughman, and J.W. Jones. 1995. Scheduling of Cool Storage Using Non-Linear Programming Techniques. IEEE Transactions on Power Systems 10(3):1279-1285.

Seem, J.E. and J.E. Braun. 1991. Adaptive Methods for Real-Time Forecasting of Building Electrical Demand. ASHRAE Transactions 97(1):710-720.

Yoshida, H. and T. Terai. 1990-1991. An ARMA Type Weather Model for Air-Conditioning, Heating and Cooling Load Calculation. Energy and Buildings 15-16:625-634.

Yoshida, H. and T. Terai. 1992. Modeling of Weather Data by Time Series Analysis for Air Conditioning Load Calculations. ASHRAE Transactions 98(1):328-345. 
